Supporting Information

\title{
Schirmer Paper Noninvasive Microsampling for Direct Mass Spectrometry Analysis of Human Tears
}

\author{
Ya-Nan Yao, ${ }^{1}$ Dandan Di, ${ }^{1}$ Zi-Cheng Yuan, ${ }^{1}$ Lin $\mathrm{Wu},{ }^{1}$ Bin $\mathrm{Hu}^{1 *}$ \\ ${ }^{1}$ Institute of Mass Spectrometry and Atmospheric Environment, Guangdong Provincial Engineering \\ Research Center for On-line Source Apportionment System of Air Pollution, Jinan University, \\ Guangzhou 510632, China
}

*Corresponding author:

Dr. Bin $\mathrm{Hu}$

Institute of Mass Spectrometry and Atmospheric Environment

Jinan University, Guangzhou 510632, China

E-mail: bin.hu@jnu.edu.cn.

Tel./Fax: +86-20-8522 5991. 


\section{Contents}

Table S1. Biomarker candidates of DED-afflicted tears obtained under positive ion detection mode.

Table S2. Biomarker candidates of DED-afflicted tears obtained under negative ion detection mode.

Figure S1. Methods for environmental exposures to human eyes.

Figure S2. Repeated Schirmer PSMS analysis of tear samples.

Figure S3. PLS-DA and PCA analysis of healthy and DED-afflicted tear samples.

Figure S4. PSMS spectra of levofloxacin, benzalkonium, and norfloxacin.

Figure S5. Standard curves of levofloxacin and benzalkonium in tear samples.

Figure S6. Schirmer PSMS analysis of eye medicines in tear samples.

Figure S7. Identification of cocaine and ketamine.

Figure S8. Identification of arginine.

Figure S9. Identification of glucose and glucose adducts.

Figure S10. Identification of salbutamol.

Figure S11. Identification of nicotine and nicotine-d4.

Figure S12. Schirmer PSMS analysis of perfume and human tear samples exposed to perfume.

Figure S13. ESI-MS analysis of perfume in methanol spiked with salts.

Figure S14. GC-MS analysis of perfume in methanol. 
Table S1. The VIP values and proposed chemical formulas of potential biomarkers of DED in human tears under positive ion detection mode. Ion species were tentatively proposed by the Human Metabolome Database (HMDB ver 4.0) (https://hmdb.ca); Unknown: not searched from the database; NA: not available. Delta $=[$ abs (experimental mass - adduct mass $) /$ adduct mass $] \times 10^{6}$.

\begin{tabular}{|c|c|c|c|c|}
\hline VIP scores & Ions $(\mathrm{m} / \mathrm{z})$ & Tentatively proposed formulae & Adducts & Delta (ppm) \\
\hline \multirow{3}{*}{2.57140} & \multirow{3}{*}{214.12004} & $\mathrm{C}_{12} \mathrm{H}_{17} \mathrm{NO}$ & {$[\mathrm{M}+\mathrm{Na}]^{+}$} & 1 \\
\hline & & $\mathrm{C}_{12} \mathrm{H}_{16} \mathrm{~F}_{3} \mathrm{~N}$ & {$\left[\mathrm{M}+\mathrm{H}-\mathrm{H}_{2} \mathrm{O}\right]^{+}$} & 3 \\
\hline & & $\mathrm{C}_{9} \mathrm{H}_{17} \mathrm{~N}_{3} \mathrm{O}_{4}$ & {$\left[\mathrm{M}+\mathrm{H}-\mathrm{H}_{2} \mathrm{O}\right]^{+}$} & 4 \\
\hline 2.43426 & 157.08348 & $\mathrm{C}_{6} \mathrm{H}_{14} \mathrm{O}_{3}$ & {$[\mathrm{M}+\mathrm{Na}]^{+}$} & 0 \\
\hline \multirow{3}{*}{2.32979} & \multirow{3}{*}{245.07825} & $\mathrm{C}_{12} \mathrm{H}_{14} \mathrm{O}_{4}$ & {$[\mathrm{M}+\mathrm{Na}]^{+}$} & 1 \\
\hline & & $\mathrm{C}_{9} \mathrm{H}_{14} \mathrm{~N}_{2} \mathrm{O}_{7}$ & {$\left[\mathrm{M}+\mathrm{H}-\mathrm{H}_{2} \mathrm{O}\right]^{+}$} & 3 \\
\hline & & $\mathrm{C}_{11} \mathrm{H}_{12} \mathrm{~N}_{4}$ & {$[\mathrm{M}+2 \mathrm{Na}-\mathrm{H}]^{+}$} & 4 \\
\hline 2.32443 & 215.12344 & Unknown & Unknown & NA \\
\hline \multirow{2}{*}{2.32043} & \multirow{2}{*}{191.10410} & $\mathrm{C}_{10} \mathrm{H}_{16} \mathrm{O}_{2}$ & {$[\mathrm{M}+\mathrm{Na}]^{+}$} & 1 \\
\hline & & $\mathrm{C}_{5} \mathrm{H}_{8} \mathrm{O}$ & {$[2 \mathrm{M}+\mathrm{Na}]^{+}$} & 1 \\
\hline 2.31778 & 217.15614 & $\mathrm{C}_{13} \mathrm{H}_{22} \mathrm{O}$ & {$[\mathrm{M}+\mathrm{Na}]^{+}$} & 1 \\
\hline \multirow{2}{*}{2.25184} & \multirow{2}{*}{169.09467} & $\mathrm{C}_{6} \mathrm{H}_{14} \mathrm{~N}_{2} \mathrm{O}_{2}$ & {$[\mathrm{M}+\mathrm{Na}]^{+}$} & 0 \\
\hline & & $\mathrm{C}_{3} \mathrm{H}_{7} \mathrm{NO}$ & {$[2 \mathrm{M}+\mathrm{Na}]^{+}$} & 0 \\
\hline \multirow{2}{*}{2.00909} & \multirow{2}{*}{376.29736} & $\mathrm{C}_{27} \mathrm{H}_{36} \mathrm{O}$ & {$\left[\mathrm{M}+\mathrm{NH}_{4}-\mathrm{H}_{2} \mathrm{O}\right]^{+}$} & 5 \\
\hline & & $\mathrm{C}_{11} \mathrm{H}_{17} \mathrm{NO}$ & {$\left[2 \mathrm{M}+\mathrm{NH}_{4}\right]^{+}$} & 4 \\
\hline \multirow{5}{*}{1.95878} & \multirow{5}{*}{147.11268} & $\mathrm{C}_{6} \mathrm{H}_{11} \mathrm{NO}_{2}$ & {$\left[\mathrm{M}+\mathrm{NH}_{4}\right]^{+}$} & 1 \\
\hline & & $\mathrm{C}_{5} \mathrm{H}_{10} \mathrm{~N}_{2} \mathrm{O}$ & {$\left[\mathrm{M}+\mathrm{CH}_{3} \mathrm{OH}+\mathrm{H}\right]^{+}$} & 1 \\
\hline & & $\mathrm{C}_{6} \mathrm{H}_{14} \mathrm{~N}_{2} \mathrm{O}_{2}$ & {$[\mathrm{M}+\mathrm{H}]^{+}$} & 1 \\
\hline & & $\mathrm{C}_{6} \mathrm{H}_{13} \mathrm{NO}_{3}$ & {$\left[\mathrm{M}+\mathrm{NH}_{4}-\mathrm{H}_{2} \mathrm{O}\right]^{+}$} & 3 \\
\hline & & $\mathrm{C}_{3} \mathrm{H}_{7} \mathrm{NO}$ & {$[2 \mathrm{M}+\mathrm{H}]^{+}$} & 1 \\
\hline 1.94887 & 185.11472 & $\mathrm{C}_{8} \mathrm{H}_{18} \mathrm{O}_{3}$ & {$[\mathrm{M}+\mathrm{Na}]^{+}$} & 0 \\
\hline \multirow{3}{*}{1.92511} & \multirow{3}{*}{301.14076} & $\mathrm{C}_{16} \mathrm{H}_{22} \mathrm{O}_{4}$ & {$[\mathrm{M}+\mathrm{Na}]^{+}$} & 1 \\
\hline & & $\mathrm{C}_{13} \mathrm{H}_{26} \mathrm{O}_{5}$ & {$[\mathrm{M}+\mathrm{K}]^{+}$} & 1 \\
\hline & & $\mathrm{C}_{12} \mathrm{H}_{16} \mathrm{~N}_{2} \mathrm{O}_{5}$ & {$\left[\mathrm{M}+\mathrm{CH}_{3} \mathrm{OH}+\mathrm{H}\right]^{+}$} & 4 \\
\hline \multirow{3}{*}{1.89818} & \multirow{3}{*}{128.14340} & $\mathrm{C}_{8} \mathrm{H}_{17} \mathrm{~N}$ & {$[\mathrm{M}+\mathrm{H}]^{+}$} & 0 \\
\hline & & $\mathrm{C}_{8} \mathrm{H}_{14}$ & {$\left[\mathrm{M}+\mathrm{NH}_{4}\right]^{+}$} & 0 \\
\hline & & $\mathrm{C}_{8} \mathrm{H}_{16} \mathrm{O}$ & {$\left[\mathrm{M}+\mathrm{NH}_{4}-\mathrm{H}_{2} \mathrm{O}\right]^{+}$} & 5 \\
\hline 1.89764 & 287.17275 & $\mathrm{C}_{11} \mathrm{H}_{18} \mathrm{~N}_{4} \mathrm{O}_{3}$ & {$\left[\mathrm{M}+\mathrm{CH}_{3} \mathrm{OH}+\mathrm{H}\right]^{+}$} & 5 \\
\hline \multirow{4}{*}{1.89564} & \multirow{4}{*}{192.13805} & $\mathrm{C}_{12} \mathrm{H}_{14} \mathrm{O}$ & {$\left[\mathrm{M}+\mathrm{NH}_{4}\right]^{+}$} & 1 \\
\hline & & $\mathrm{C}_{12} \mathrm{H}_{17} \mathrm{NO}$ & {$[\mathrm{M}+\mathrm{H}]^{+}$} & 1 \\
\hline & & $\mathrm{C}_{11} \mathrm{H}_{13} \mathrm{~N}$ & {$\left[\mathrm{M}+\mathrm{CH}_{3} \mathrm{OH}+\mathrm{H}\right]^{+}$} & 1 \\
\hline & & $\mathrm{C}_{12} \mathrm{H}_{16} \mathrm{O}_{2}$ & {$\left[\mathrm{M}+\mathrm{NH}_{4}-\mathrm{H}_{2} \mathrm{O}\right]^{+}$} & 2 \\
\hline 1.89144 & 193.14148 & Unknown & Unknown & NA \\
\hline \multirow{4}{*}{1.88821} & \multirow{4}{*}{136.11200} & $\mathrm{C}_{9} \mathrm{H}_{10}$ & {$\left[\mathrm{M}+\mathrm{NH}_{4}\right]^{+}$} & 1 \\
\hline & & $\mathrm{C}_{9} \mathrm{H}_{13} \mathrm{~N}$ & {$[\mathrm{M}+\mathrm{H}]^{+}$} & 1 \\
\hline & & $\mathrm{C}_{9} \mathrm{H}_{12} \mathrm{O}$ & {$\left[\mathrm{M}+\mathrm{NH}_{4}-\mathrm{H}_{2} \mathrm{O}\right]^{+}$} & 4 \\
\hline & & $\mathrm{C}_{9} \mathrm{H}_{15} \mathrm{NO}$ & {$\left[\mathrm{M}+\mathrm{H}-\mathrm{H}_{2} \mathrm{O}\right]^{+}$} & 5 \\
\hline \multirow{3}{*}{1.87930} & \multirow{3}{*}{279.15873} & $\mathrm{C}_{15} \mathrm{H}_{18} \mathrm{O}_{3}$ & {$\left[\mathrm{M}+\mathrm{CH}_{3} \mathrm{OH}+\mathrm{H}\right]^{+}$} & 1 \\
\hline & & $\mathrm{C}_{16} \mathrm{H}_{22} \mathrm{O}_{4}$ & {$[\mathrm{M}+\mathrm{H}]^{+}$} & 1 \\
\hline & & $\mathrm{C}_{16} \mathrm{H}_{24} \mathrm{O}_{5}$ & {$\left[\mathrm{M}+\mathrm{H}-\mathrm{H}_{2} \mathrm{O}\right]^{+}$} & 3 \\
\hline \multirow{3}{*}{1.87258} & \multirow{3}{*}{163.13270} & $\mathrm{C}_{7} \mathrm{H}_{14} \mathrm{O}_{2}$ & {$\left[\mathrm{M}+\mathrm{CH}_{3} \mathrm{OH}+\mathrm{H}\right]^{+}$} & 1 \\
\hline & & $\mathrm{C}_{8} \mathrm{H}_{18} \mathrm{O}_{3}$ & {$[\mathrm{M}+\mathrm{H}]^{+}$} & 1 \\
\hline & & $\mathrm{C}_{4} \mathrm{H}_{10} \mathrm{O}_{2}$ & {$\left[2 \mathrm{M}+\mathrm{H}-\mathrm{H}_{2} \mathrm{O}\right]^{+}$} & 1 \\
\hline
\end{tabular}


Table S1. Continued.

\begin{tabular}{|c|c|c|c|c|}
\hline VIP scores & Ions $(m / z)$ & Tentatively proposed formulae & Adducts & Delta (ppm) \\
\hline \multirow{4}{*}{1.84294} & \multirow{4}{*}{165.13848} & $\mathrm{C}_{10} \mathrm{H}_{16} \mathrm{NO}$ & {$\left[\mathrm{M}+\mathrm{NH}_{4}-\mathrm{H}_{2} \mathrm{O}\right]^{+}$} & 1 \\
\hline & & $\mathrm{C}_{10} \mathrm{H}_{13} \mathrm{~N}$ & {$\left[\mathrm{M}+\mathrm{NH}_{4}\right]^{+}$} & 1 \\
\hline & & $\mathrm{C}_{10} \mathrm{H}_{16} \mathrm{~N}_{2}$ & {$[\mathrm{M}+\mathrm{H}]^{+}$} & 1 \\
\hline & & $\mathrm{C}_{10} \mathrm{H}_{15} \mathrm{NO}$ & {$\left[\mathrm{M}+\mathrm{NH}_{4}-\mathrm{H}_{2} \mathrm{O}\right]^{+}$} & 3 \\
\hline \multirow{2}{*}{1.83803} & \multirow{2}{*}{158.15381} & $\mathrm{C}_{9} \mathrm{H}_{16} \mathrm{O}$ & {$\left[\mathrm{M}+\mathrm{NH}_{4}\right]^{+}$} & 1 \\
\hline & & $\mathrm{C}_{9} \mathrm{H}_{18} \mathrm{O}_{2}$ & {$\left[\mathrm{M}+\mathrm{NH}_{4}-\mathrm{H}_{2} \mathrm{O}\right]^{+}$} & 3 \\
\hline 1.82657 & 171.09903 & $\mathrm{C}_{5} \mathrm{H}_{7} \mathrm{~N}_{5} \mathrm{O}$ & {$\left[\mathrm{M}+\mathrm{NH}_{4}\right]^{+}$} & 1 \\
\hline \multirow{2}{*}{1.60513} & \multirow{2}{*}{467.10167} & $\mathrm{C}_{25} \mathrm{H}_{26} \mathrm{O}_{4}$ & {$[\mathrm{M}+2 \mathrm{~K}-\mathrm{H}]^{+}$} & 1 \\
\hline & & $\mathrm{C}_{9} \mathrm{H}_{18} \mathrm{~S}_{3}$ & {$[2 \mathrm{M}+\mathrm{Na}]^{+}$} & 4 \\
\hline \multirow{5}{*}{1.50793} & \multirow{2}{*}{177.16361} & $\mathrm{C}_{13} \mathrm{H}_{20}$ & {$[\mathrm{M}+\mathrm{H}]^{+}$} & 1 \\
\hline & & $\mathrm{C}_{13} \mathrm{H}_{22} \mathrm{O}$ & {$\left[\mathrm{M}+\mathrm{H}-\mathrm{H}_{2} \mathrm{O}\right]^{+}$} & 4 \\
\hline & \multirow{3}{*}{195.17414} & $\mathrm{C}_{12} \mathrm{H}_{18}$ & {$\left[\mathrm{M}+\mathrm{CH}_{3} \mathrm{OH}+\mathrm{H}\right]^{+}$} & 1 \\
\hline & & $\mathrm{C}_{13} \mathrm{H}_{22} \mathrm{O}$ & {$[\mathrm{M}+\mathrm{H}]^{+}$} & 1 \\
\hline & & $\mathrm{C}_{13} \mathrm{H}_{24} \mathrm{O}_{2}$ & {$\left[\mathrm{M}+\mathrm{H}-\mathrm{H}_{2} \mathrm{O}\right]^{+}$} & 4 \\
\hline \multirow{3}{*}{1.50790} & \multirow{3}{*}{153.12724} & $\mathrm{C}_{9} \mathrm{H}_{12}$ & {$\left[\mathrm{M}+\mathrm{CH}_{3} \mathrm{OH}+\mathrm{H}\right]^{+}$} & 1 \\
\hline & & $\mathrm{C}_{10} \mathrm{H}_{16} \mathrm{O}$ & {$[\mathrm{M}+\mathrm{H}]^{+}$} & 1 \\
\hline & & $\mathrm{C}_{10} \mathrm{H}_{18} \mathrm{O}_{2}$ & {$\left[\mathrm{M}+\mathrm{H}-\mathrm{H}_{2} \mathrm{O}\right]^{+}$} & 5 \\
\hline 1.50340 & 149.02322 & $\mathrm{C}_{8} \mathrm{H}_{6} \mathrm{O}_{4}$ & {$\left[\mathrm{M}+\mathrm{H}-\mathrm{H}_{2} \mathrm{O}\right]^{+}$} & 5 \\
\hline \multirow{2}{*}{1.50302} & \multirow{2}{*}{137.13236} & $\mathrm{C}_{10} \mathrm{H}_{16}$ & {$[\mathrm{M}+\mathrm{H}]^{+}$} & 1 \\
\hline & & $\mathrm{C}_{10} \mathrm{H}_{18} \mathrm{O}$ & {$\left[\mathrm{M}+\mathrm{H}-\mathrm{H}_{2} \mathrm{O}\right]^{+}$} & 5 \\
\hline \multirow{3}{*}{1.48615} & \multirow{3}{*}{285.16094} & $\mathrm{C}_{17} \mathrm{H}_{26} \mathrm{O}$ & {$[\mathrm{M}+\mathrm{K}]^{+}$} & 2 \\
\hline & & $\mathrm{C}_{17} \mathrm{H}_{20} \mathrm{~N}_{2} \mathrm{O}_{2}$ & {$[\mathrm{M}+\mathrm{H}]^{+}$} & 4 \\
\hline & & $\mathrm{C}_{17} \mathrm{H}_{17} \mathrm{NO}_{2}$ & {$\left[\mathrm{M}+\mathrm{NH}_{4}\right]^{+}$} & 4 \\
\hline \multirow{2}{*}{1.47921} & \multirow{2}{*}{307.13746} & $\mathrm{C}_{5} \mathrm{H}_{7} \mathrm{~N}_{5} \mathrm{O}$ & {$[2 \mathrm{M}+\mathrm{H}]^{+}$} & 0 \\
\hline & & $\mathrm{C}_{9} \mathrm{H}_{15} \mathrm{~N}_{5} \mathrm{O}_{6}$ & {$\left[\mathrm{M}+\mathrm{NH}_{4}\right]^{+}$} & 5 \\
\hline 1.31409 & 205.06822 & $\mathrm{C}_{6} \mathrm{H}_{14} \mathrm{O}_{6}$ & {$[\mathrm{M}+\mathrm{Na}]^{+}$} & 0 \\
\hline 1.29455 & 175.10928 & $\mathrm{C}_{10} \mathrm{H}_{16} \mathrm{O}$ & {$[\mathrm{M}+\mathrm{Na}]^{+}$} & 0 \\
\hline \multirow{8}{*}{1.18580} & \multirow{5}{*}{150.09123} & $\mathrm{C}_{9} \mathrm{H}_{11} \mathrm{NO}$ & {$[\mathrm{M}+\mathrm{H}]^{+}$} & 1 \\
\hline & & $\mathrm{C}_{9} \mathrm{H}_{8} \mathrm{O}$ & {$\left[\mathrm{M}+\mathrm{NH}_{4}\right]^{+}$} & 1 \\
\hline & & $\mathrm{C}_{8} \mathrm{H}_{7} \mathrm{~N}$ & {$\left[\mathrm{M}+\mathrm{CH}_{3} \mathrm{OH}+\mathrm{H}\right]^{+}$} & 1 \\
\hline & & $\mathrm{C}_{9} \mathrm{H}_{10} \mathrm{O}_{2}$ & {$\left[\mathrm{M}+\mathrm{NH}_{4}-\mathrm{H}_{2} \mathrm{O}\right]^{+}$} & 3 \\
\hline & & $\mathrm{C}_{9} \mathrm{H}_{13} \mathrm{NO}_{2}$ & {$\left[\mathrm{M}+\mathrm{H}-\mathrm{H}_{2} \mathrm{O}\right]^{+}$} & 5 \\
\hline & 166.08388 & $\mathrm{C}_{7} \mathrm{H}_{13} \mathrm{NO}_{2}$ & {$[\mathrm{M}+\mathrm{Na}]^{+}$} & 0 \\
\hline & 168.10756 & Unknown & Unknown & NA \\
\hline & 180.10749 & Unknown & Unknown & NA \\
\hline \multirow{3}{*}{1.18567} & \multirow{3}{*}{104.10735} & $\mathrm{C}_{5} \mathrm{H}_{14} \mathrm{NO}$ & {$[\mathrm{M}+\mathrm{H}]^{+}$} & 2 \\
\hline & & $\mathrm{C}_{5} \mathrm{H}_{10} \mathrm{O}$ & {$\left[\mathrm{M}+\mathrm{NH}_{4}\right]^{+}$} & 3 \\
\hline & & $\mathrm{C}_{4} \mathrm{H}_{9} \mathrm{~N}$ & {$\left[\mathrm{M}+\mathrm{CH}_{3} \mathrm{OH}+\mathrm{H}\right]^{+}$} & 3 \\
\hline \multirow{2}{*}{1.15155} & \multirow{2}{*}{205.19487} & $\mathrm{C}_{15} \mathrm{H}_{24}$ & {$[\mathrm{M}+\mathrm{H}]^{+}$} & 1 \\
\hline & & $\mathrm{C}_{15} \mathrm{H}_{26} \mathrm{O}$ & {$\left[\mathrm{M}+\mathrm{H}-\mathrm{H}_{2} \mathrm{O}\right]^{+}$} & 4 \\
\hline
\end{tabular}


Table S1. Continued.

\begin{tabular}{|c|c|c|c|c|}
\hline $\begin{array}{l}\text { VIP } \\
\text { scores }\end{array}$ & Ions $(m / z)$ & Tentatively proposed formulae & Adducts & Delta (ppm) \\
\hline \multirow{6}{*}{1.14367} & 146.06616 & Unknown & Unknown & NA \\
\hline & \multirow{5}{*}{167.10421} & $\mathrm{C}_{8} \mathrm{H}_{16} \mathrm{O}_{2}$ & {$[\mathrm{M}+\mathrm{Na}]^{+}$} & 0 \\
\hline & & $\mathrm{C}_{4} \mathrm{H}_{8} \mathrm{O}$ & {$[2 \mathrm{M}+\mathrm{Na}]^{+}$} & 0 \\
\hline & & $\mathrm{C}_{6} \mathrm{H}_{10} \mathrm{~N}_{6}$ & {$[\mathrm{M}+\mathrm{H}]^{+}$} & 1 \\
\hline & & $\mathrm{C}_{3} \mathrm{H}_{5} \mathrm{~N}_{3}$ & {$[2 \mathrm{M}+\mathrm{H}]^{+}$} & 1 \\
\hline & & $\mathrm{C}_{6} \mathrm{H}_{7} \mathrm{~N}_{5}$ & {$\left[\mathrm{M}+\mathrm{NH}_{4}\right]^{+}$} & 1 \\
\hline 1.13189 & 365.10522 & $\mathrm{C}_{12} \mathrm{H}_{22} \mathrm{O}_{11}$ & {$[\mathrm{M}+\mathrm{Na}]^{+}$} & 1 \\
\hline \multirow{5}{*}{1.09733} & 138.90650 & Unknown & Unknown & NA \\
\hline & 149.11711 & $\mathrm{C}_{6} \mathrm{H}_{12} \mathrm{O}_{2}$ & {$\left[\mathrm{M}+\mathrm{CH}_{3} \mathrm{OH}+\mathrm{H}\right]^{+}$} & 1 \\
\hline & \multirow{2}{*}{154.08381} & $\mathrm{C}_{6} \mathrm{H}_{13} \mathrm{NO}_{2}$ & {$[\mathrm{M}+\mathrm{Na}]^{+}$} & 0 \\
\hline & & $\mathrm{C}_{6} \mathrm{H}_{14} \mathrm{NO}_{2}$ & {$[\mathrm{M}+\mathrm{Na}]^{+}$} & 4 \\
\hline & 169.98584 & $\mathrm{C}_{4} \mathrm{H}_{5} \mathrm{NO}_{4}$ & {$[\mathrm{M}+\mathrm{K}]^{+}$} & 5 \\
\hline 1.03839 & 177.08851 & $\mathrm{C}_{9} \mathrm{H}_{14} \mathrm{O}_{2}$ & {$[\mathrm{M}+\mathrm{Na}]^{+}$} & 0 \\
\hline
\end{tabular}


Table S2. The VIP values and proposed chemical formulas of potential biomarkers of DED in human tears under negative ion detection mode. Ion species were tentatively proposed by the Human Metabolome Database (HMDB ver 4.0). Unknown: not searched from the database; NA: not available. Delta $=[$ abs (experimental mass - adduct mass $) /$ adduct mass $] \times 10^{6}$.

\begin{tabular}{|c|c|c|c|c|}
\hline VIP scores & Ions $(m / z)$ & Tentatively proposed formulae & Adducts & Delta (ppm) \\
\hline 3.1114 & 171.13810 & Unknown & Unknown & NA \\
\hline 3.03756 & 143.10664 & $\mathrm{C}_{8} \mathrm{H}_{18} \mathrm{O}_{3}$ & {$\left[\mathrm{M}-\mathrm{H}_{2} \mathrm{O}-\mathrm{H}\right]^{-}$} & 4 \\
\hline 3.02637 & 157.12239 & Unknown & Unknown & NA \\
\hline 3.02623 & 241.21694 & $\mathrm{C}_{15} \mathrm{H}_{30} \mathrm{O}_{2}$ & {$[\mathrm{M}-\mathrm{H}]^{-}$} & 2 \\
\hline 2.99212 & 171.10181 & $\mathrm{C}_{9} \mathrm{H}_{16} \mathrm{O}_{3}$ & {$[\mathrm{M}-\mathrm{H}]^{-}$} & 5 \\
\hline \multirow{4}{*}{2.83073} & \multirow{4}{*}{199.16966} & $\mathrm{C}_{12} \mathrm{H}_{26} \mathrm{O}_{3}$ & {$\left[\mathrm{M}-\mathrm{H}_{2} \mathrm{O}-\mathrm{H}\right]^{-}$} & 1 \\
\hline & & $\mathrm{C}_{6} \mathrm{H}_{12} \mathrm{O}$ & {$[2 \mathrm{M}-\mathrm{H}]^{-}$} & 3 \\
\hline & & $\mathrm{C}_{12} \mathrm{H}_{24} \mathrm{O}_{2}$ & {$[\mathrm{M}-\mathrm{H}]^{-}$} & 3 \\
\hline & & $\mathrm{C}_{6} \mathrm{H}_{12} \mathrm{O}$ & {$[2 \mathrm{M}-\mathrm{H}]^{-}$} & 3 \\
\hline \multirow{3}{*}{2.6785} & \multirow{3}{*}{227.20119} & $\mathrm{C}_{14} \mathrm{H}_{30} \mathrm{O}_{3}$ & {$\left[\mathrm{M}-\mathrm{H}_{2} \mathrm{O}-\mathrm{H}\right]^{-}$} & 0 \\
\hline & & $\mathrm{C}_{14} \mathrm{H}_{28} \mathrm{O}_{2}$ & {$[\mathrm{M}-\mathrm{H}]^{-}$} & 2 \\
\hline & & $\mathrm{C}_{7} \mathrm{H}_{14} \mathrm{O}$ & {$[2 \mathrm{M}-\mathrm{H}]^{-}$} & 2 \\
\hline 2.52625 & 129.09087 & Unknown & Unknown & NA \\
\hline \multirow{2}{*}{2.21014} & \multirow{2}{*}{281.24845} & $\mathrm{C}_{18} \mathrm{H}_{34} \mathrm{O}_{2}$ & {$[\mathrm{M}-\mathrm{H}]^{-}$} & 1 \\
\hline & & $\mathrm{C}_{18} \mathrm{H}_{36} \mathrm{O}_{3}$ & {$\left[\mathrm{M}-\mathrm{H}_{2} \mathrm{O}-\mathrm{H}\right]^{-}$} & 1 \\
\hline 1.72047 & 330.04467 & Unknown & Unknown & NA \\
\hline 1.59142 & 221.14972 & Unknown & Unknown & NA \\
\hline \multirow{2}{*}{1.53398} & 166.02621 & Unknown & Unknown & NA \\
\hline & 239.05940 & $\mathrm{C}_{12} \mathrm{H}_{14} \mathrm{~N}_{2} \mathrm{O}$ & {$[\mathrm{M}+\mathrm{K}-2 \mathrm{H}]^{-}$} & 1 \\
\hline 1.48619 & 220.14637 & $\mathrm{C}_{12} \mathrm{H}_{19} \mathrm{~N}_{3} \mathrm{O}$ & {$[\mathrm{M}-\mathrm{H}]^{-}$} & 4 \\
\hline \multirow{2}{*}{1.44758} & \multirow{2}{*}{283.26419} & $\mathrm{C}_{9} \mathrm{H}_{18} \mathrm{O}$ & {$[2 \mathrm{M}-\mathrm{H}]^{-}$} & 0 \\
\hline & & $\mathrm{C}_{18} \mathrm{H}_{36} \mathrm{O}_{2}$ & {$[\mathrm{M}-\mathrm{H}]^{-}$} & 0 \\
\hline 1.42533 & 297.04693 & $\mathrm{C}_{11} \mathrm{H}_{11} \mathrm{~F}_{3} \mathrm{~N}_{2} \mathrm{O}_{3}$ & {$[\mathrm{M}+\mathrm{Na}-2 \mathrm{H}]^{-}$} & 0 \\
\hline 1.26106 & 377.08566 & $\mathrm{C}_{16} \mathrm{H}_{20} \mathrm{O}_{9}$ & {$[\mathrm{M}+\mathrm{Na}-2 \mathrm{H}]^{-}$} & 1 \\
\hline 1.24355 & 331.06126 & $\mathrm{C}_{20} \mathrm{H}_{12} \mathrm{O}_{5}$ & {$[\mathrm{M}-\mathrm{H}]^{-}$} & 0 \\
\hline \multirow{5}{*}{1.21529} & \multirow{2}{*}{253.21717} & $\mathrm{C}_{16} \mathrm{H}_{30} \mathrm{O}_{2}$ & {$[\mathrm{M}-\mathrm{H}]^{-}$} & 1 \\
\hline & & $\mathrm{C}_{16} \mathrm{H}_{32} \mathrm{O}_{3}$ & {$\left[\mathrm{M}-\mathrm{H}_{2} \mathrm{O}-\mathrm{H}\right]^{-}$} & 2 \\
\hline & \multirow{3}{*}{279.23281} & $\mathrm{C}_{18} \mathrm{H}_{32} \mathrm{O}_{2}$ & {$[\mathrm{M}-\mathrm{H}]^{-}$} & 1 \\
\hline & & $\mathrm{C}_{9} \mathrm{H}_{16} \mathrm{O}$ & {$[2 \mathrm{M}-\mathrm{H}]^{-}$} & 1 \\
\hline & & $\mathrm{C}_{18} \mathrm{H}_{34} \mathrm{O}_{3}$ & {$\left[\mathrm{M}-\mathrm{H}_{2} \mathrm{O}-\mathrm{H}\right]^{-}$} & 1 \\
\hline 1.18507 & 155.02590 & Unknown & Unknown & NA \\
\hline \multirow{2}{*}{1.11836} & \multirow{2}{*}{255.23280} & $\mathrm{C}_{8} \mathrm{H}_{16} \mathrm{O}$ & {$[2 \mathrm{M}-\mathrm{H}]^{-}$} & 1 \\
\hline & & $\mathrm{C}_{16} \mathrm{H}_{32} \mathrm{O}_{2}$ & {$[\mathrm{M}-\mathrm{H}]^{-}$} & 1 \\
\hline \multirow{2}{*}{1.08078} & \multirow{2}{*}{165.03989} & $\mathrm{C}_{4} \mathrm{H}_{8} \mathrm{~N}_{4} \mathrm{O}_{2}$ & {$[\mathrm{M}+\mathrm{Na}-2 \mathrm{H}]^{-}$} & 3 \\
\hline & & $\mathrm{C}_{5} \mathrm{H}_{10} \mathrm{O}_{6}$ & {$[\mathrm{M}-\mathrm{H}]^{-}$} & 3 \\
\hline
\end{tabular}




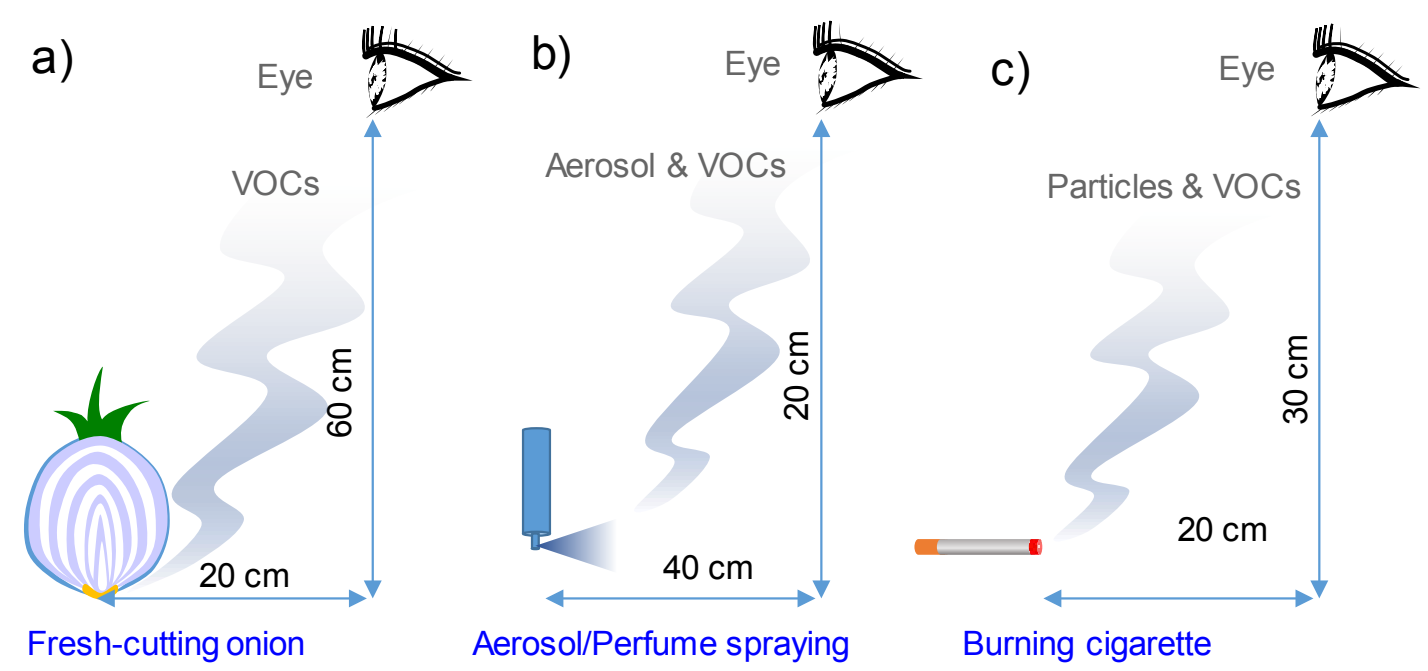

Figure S1. Environmental exposures to human eyes: a) VOCs generating from fresh-cutting onion in the air, b) aerosol/perfume spraying in the air, c) tabacoo smoke of burning cigarette in the air. 

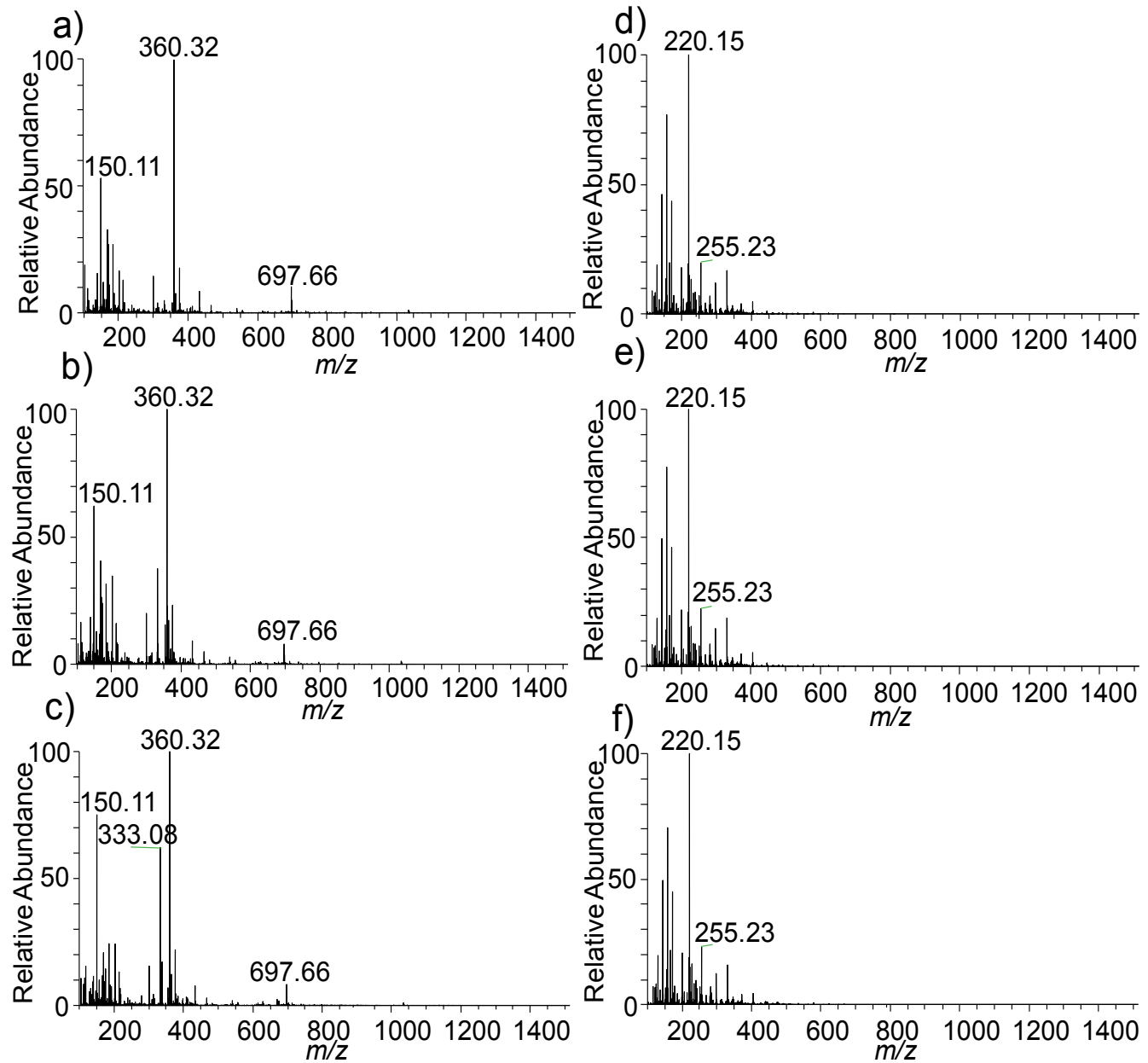

Figure S2. Repeated PSMS analysis of healthy human tears under positive ion detection mode (left column, a-c) and under negative ion detection mode (right column, d-f). The RSD values were measured by the intensities of each base peak. 
a)
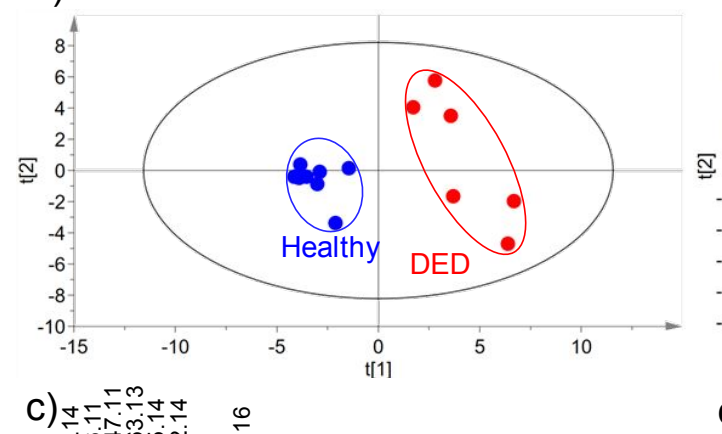

b)

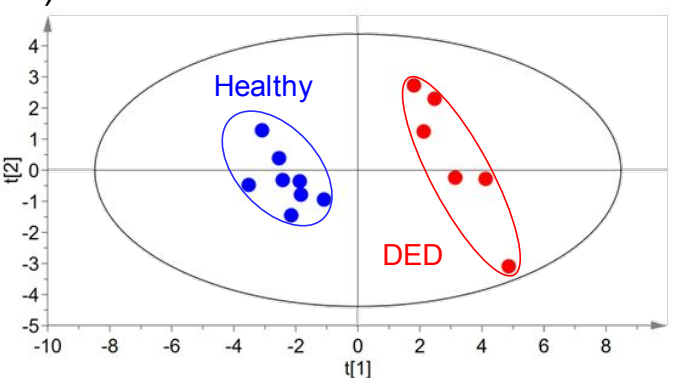

d)

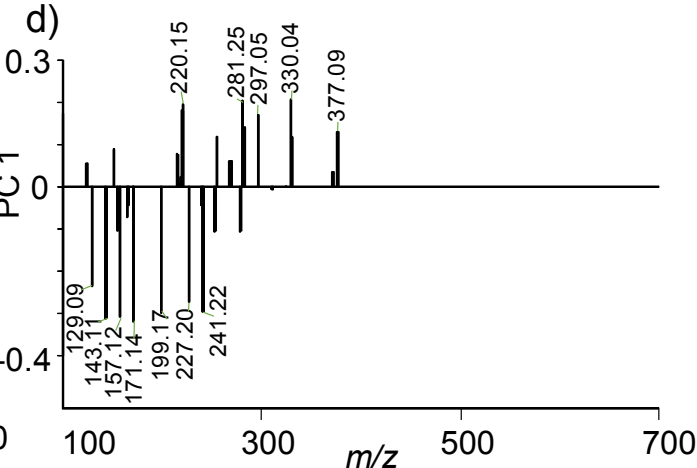

700

e)

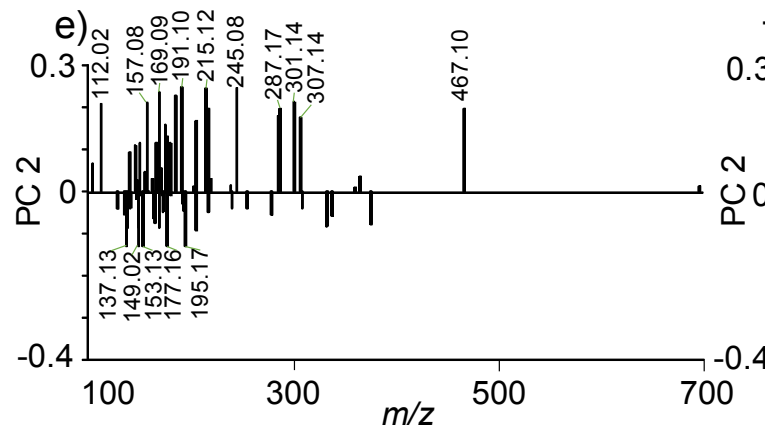

f)

)

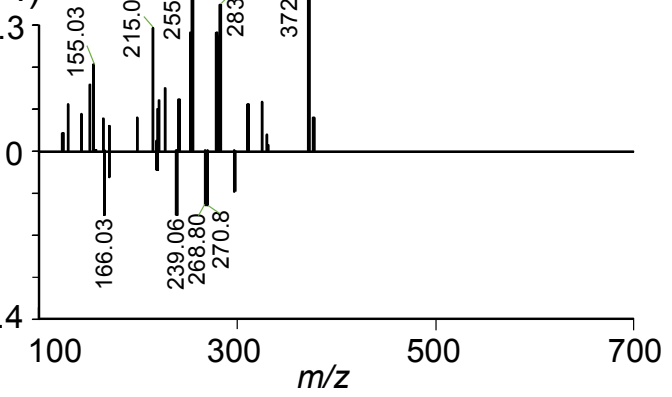

Figure S3. Statistical analysis of healthy and DED-afflicted tears: a) PLS-DA plot of healthy and DED-afflicted human tears in positive ion detection mode, b) PLS-DA plot of healthy and DEDafflicted human tears in negative ion detection mode; c) PC1 loading plot in positive ion detection mode, d) loading plot of PC1 in negative ion detection mode, e) PC2 loading plot in positive ion detection mode, f) loading plot of PC2 in negative ion detection mode. The peaks in c-f) were tentatively proposed in Table S1 and Table S2. 

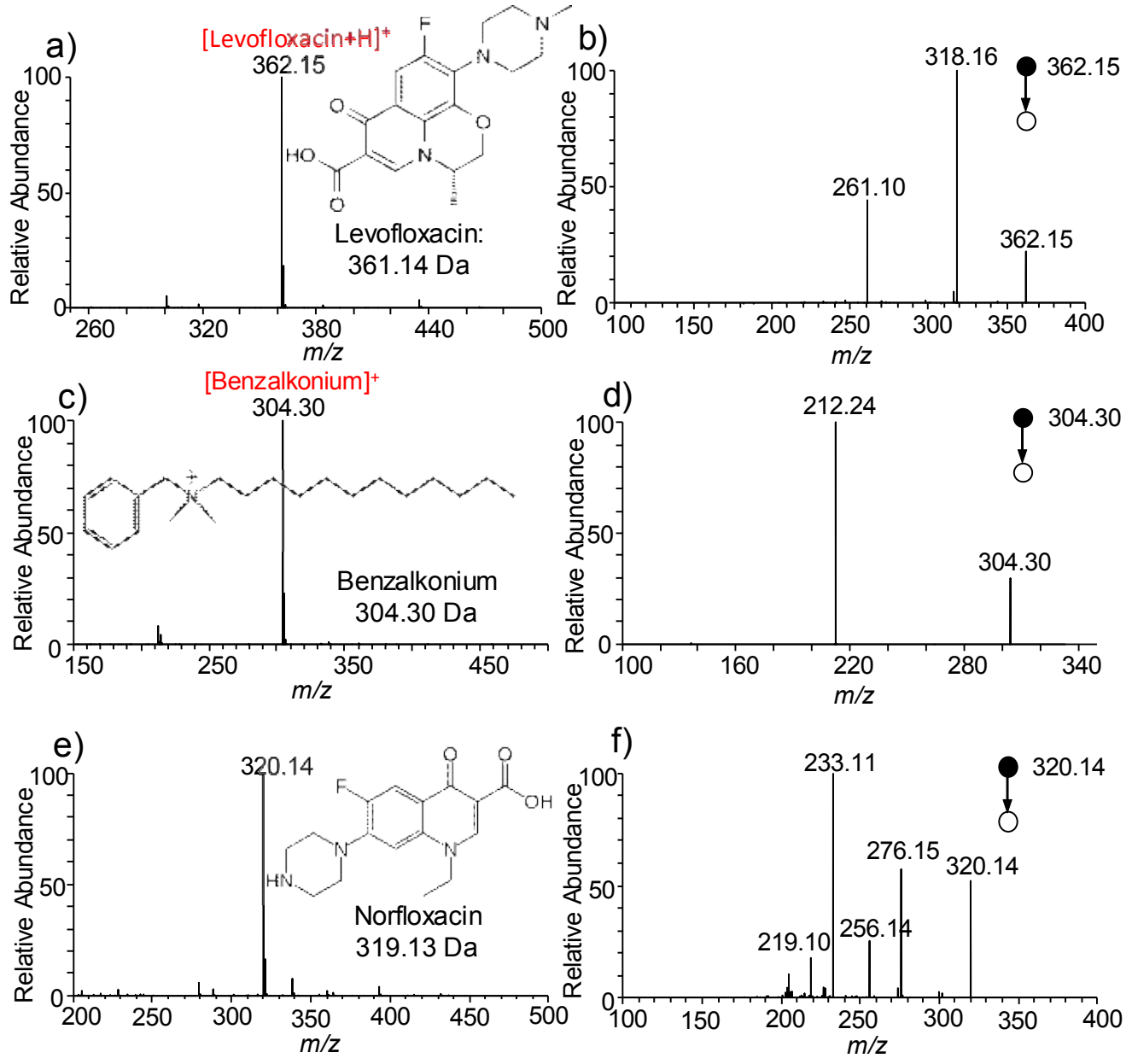

Figure S4. PSMS spectra of authorized compounds: a) mass spectrum of levofloxacin, b) MS/MS spectrum of protonated levofloxacin $(\mathrm{m} / \mathrm{z} 362.15)$, c) mass spectrum of benzalkonium, d) MS/MS spectrum of benzalkonium cation ( $\mathrm{m} / \mathrm{z} 304.30)$, e) MS/MS spectrum of norfloxacin, f) MS/MS spectrum of protonated norfloxacin $(\mathrm{m} / \mathrm{z} 320.14)$. 

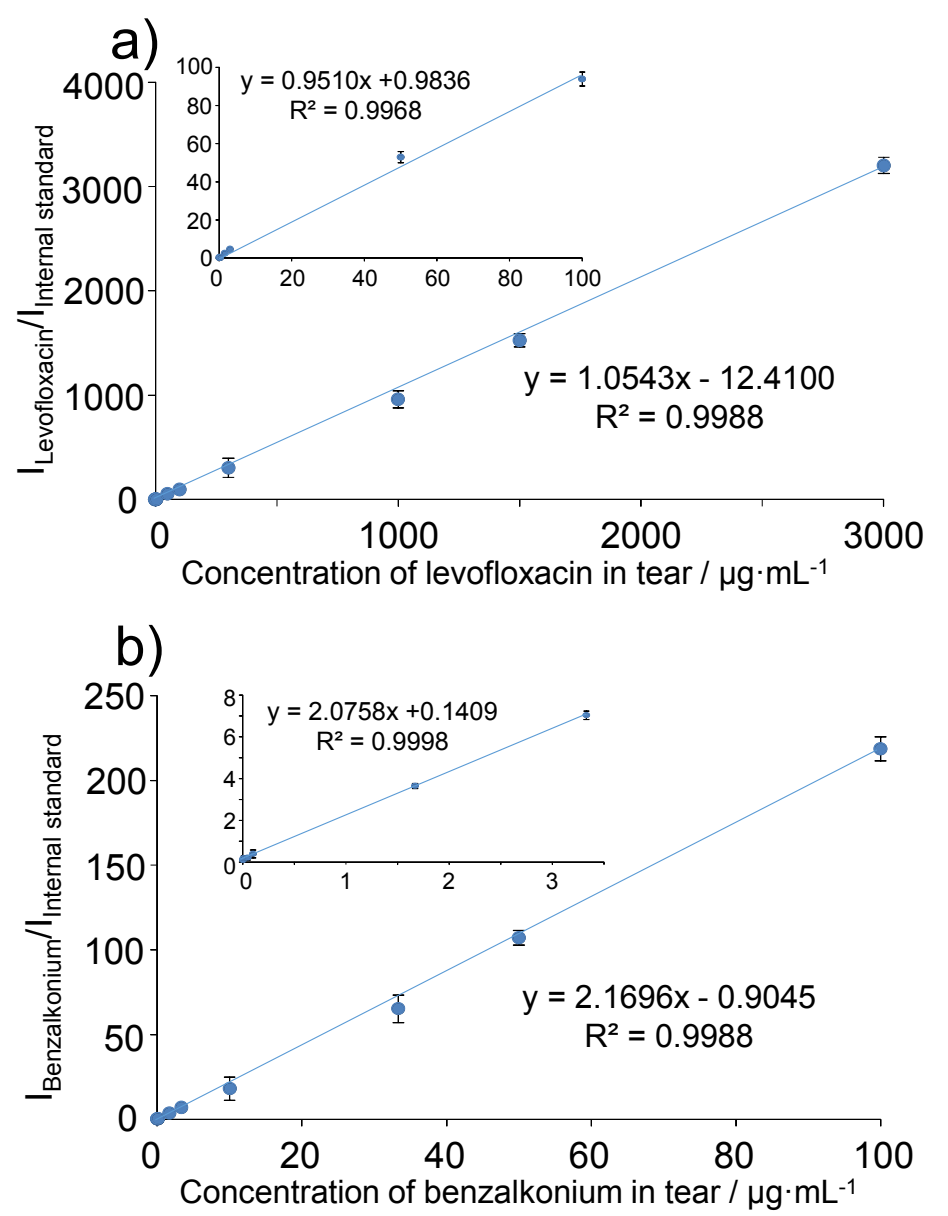

Figure S5. a) Plots of the signal ratio of levofloxacin/norfloxacin against the levofloxacin concentration in human tear samples; b) Plot of the signal ratio of benzalkonium/norfloxacin against the benzalkonium concentrations in human tear samples. Insets show the curves obtained at low concentrations. 

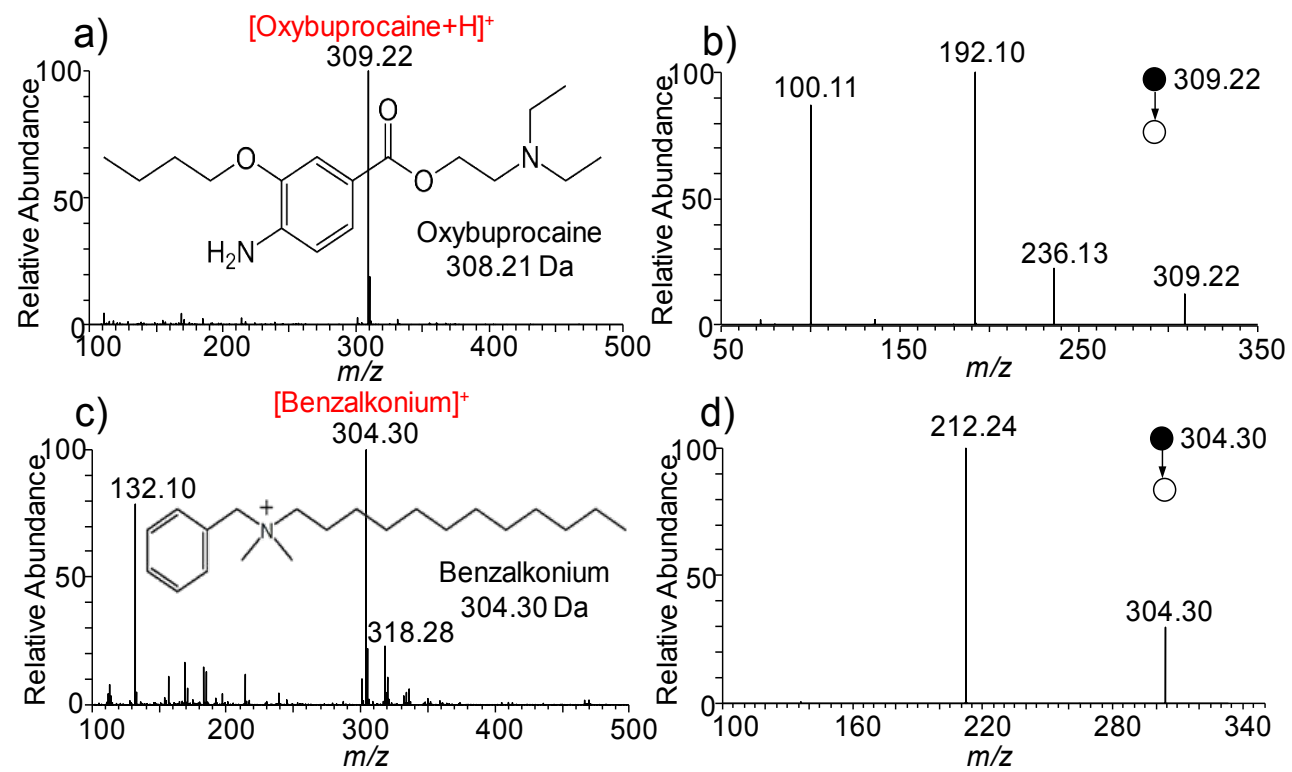

Figure S6. Schirmer paper sampling and PSMS analysis of human tear samples after dropping different eye medicines: a) Oxybuprocaine hydrochloride eye drops, b) MS/MS spectrum of protonated oxybuprocaine at $\mathrm{m} / \mathrm{z} 309.22$, c) Sodium hyaluronate eye drops, d) MS/MS spectrum of radical cation of benzalkonium at $m / z 304.30$. 

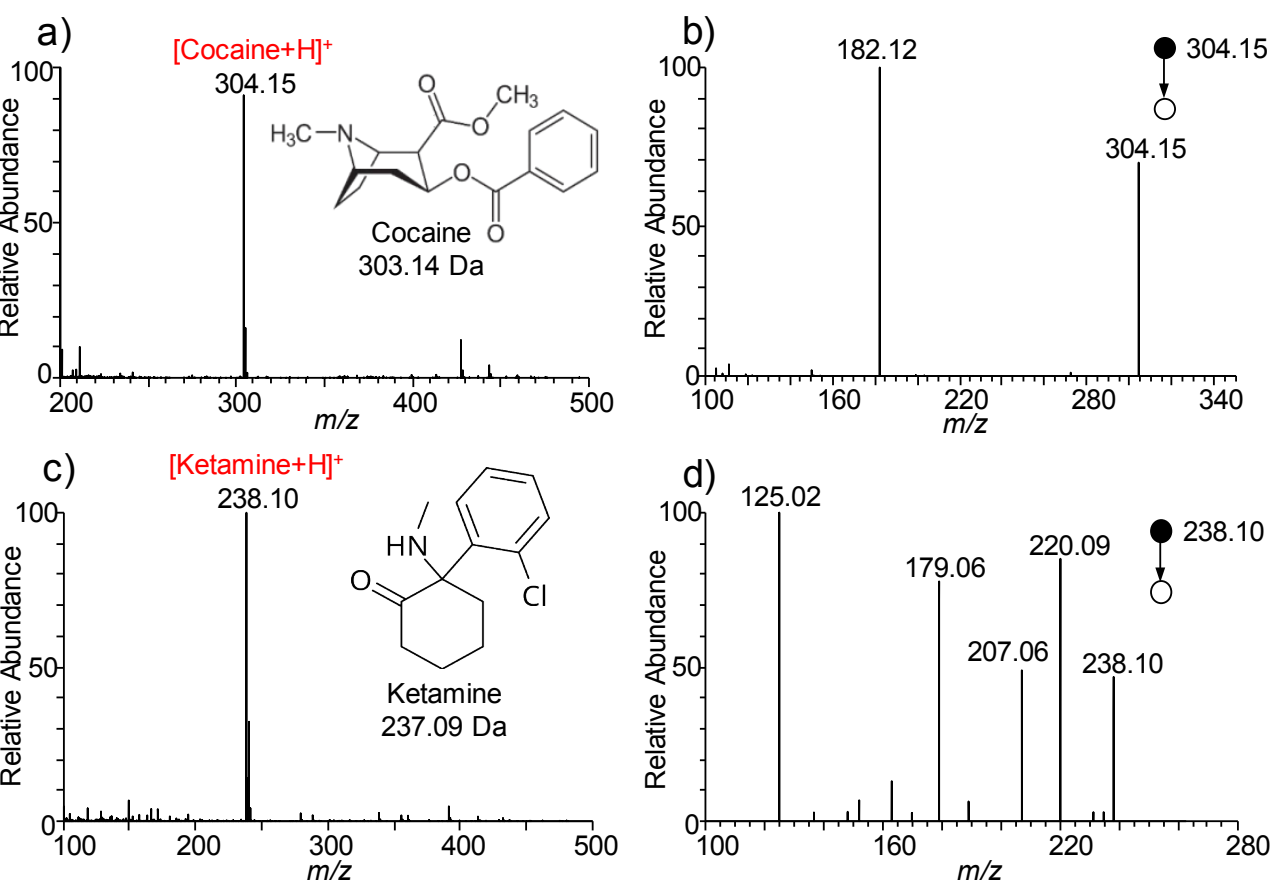

Figure S7. PSMS spectra of authorized compounds: a) mass spectrum of cocaine, b) MS/MS spectrum of cocaine $(\mathrm{m} / \mathrm{z} 304.15)$, c) mass spectrum of ketamine, d) MS/MS spectrum of protonated ketamine $(m / z 238.10)$. 

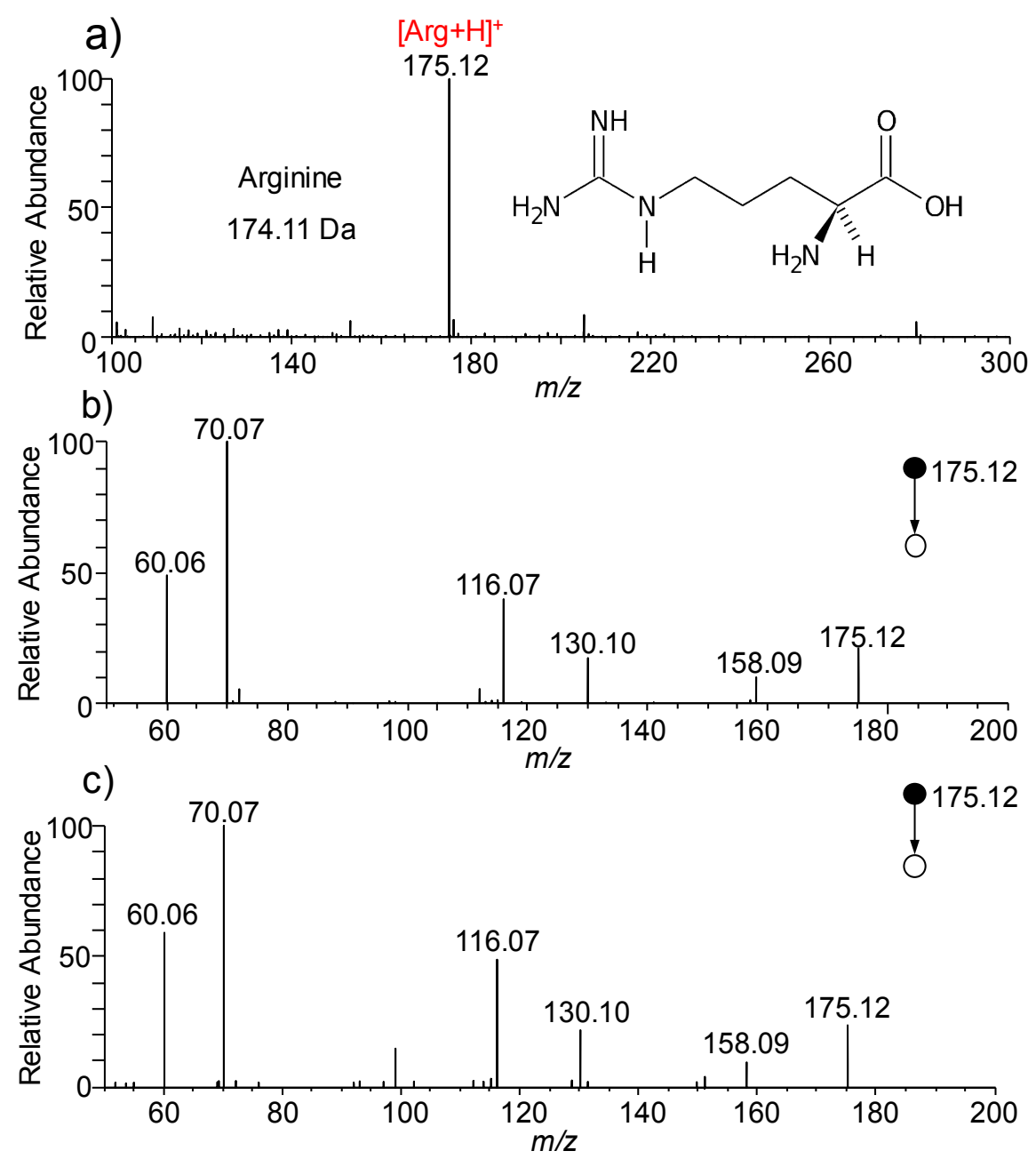

Figure S8. a) PSMS spectrum of authorized arginine, b) PSMS/MS spectrum of protonated arginine at $m / z$ 175.12, c) PSMS/MS spectrum of the ions at $m / z 175.12$ from Figure $4 \mathrm{a}$. 

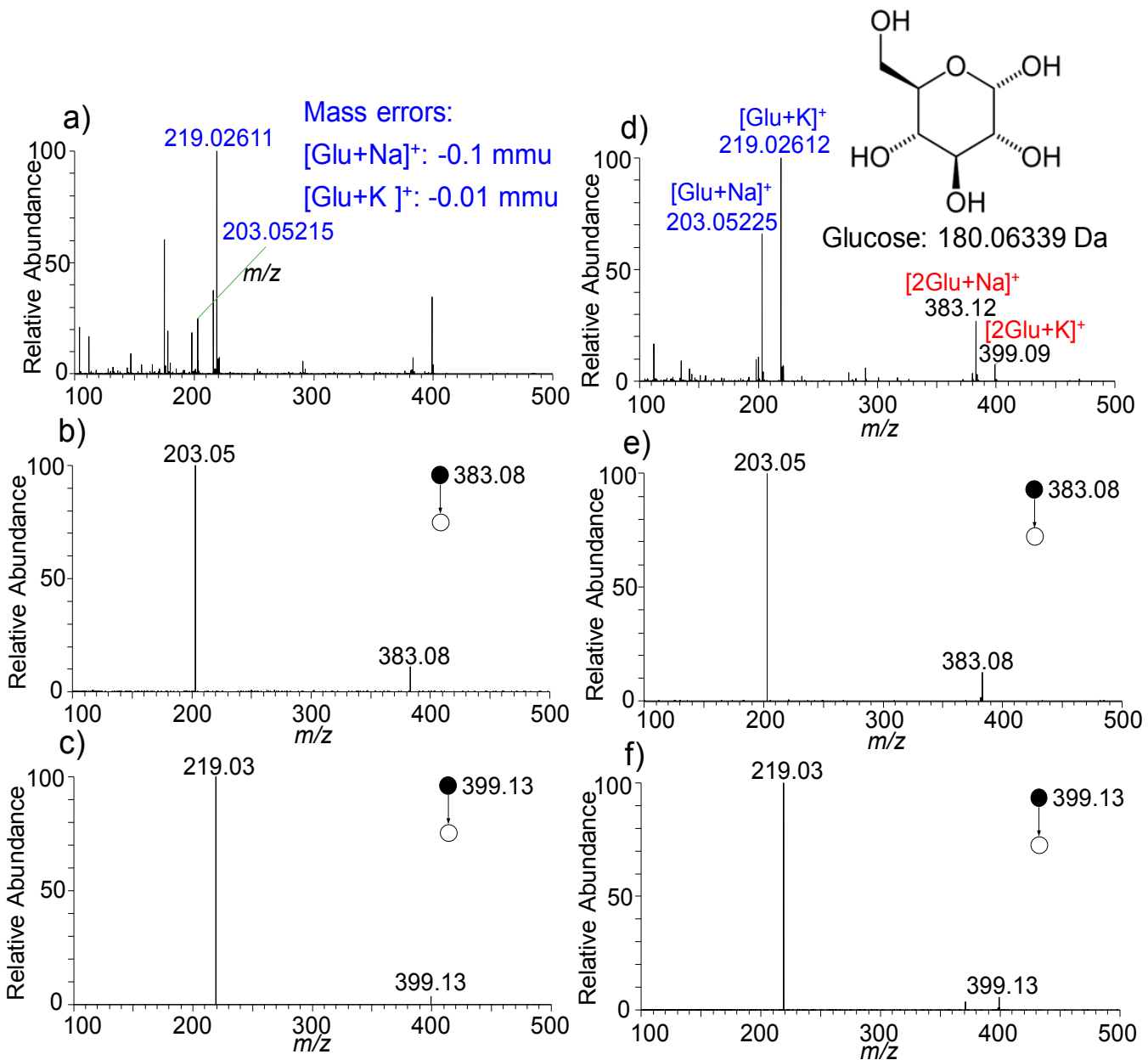

Figure S9. a) PSMS spectrum obtained by direct analysis of human tears exposed to fresh-cutting onions (same to Figure 4a); b) PSMS/MS spectrum of ions at $\mathrm{m} / \mathrm{z} 383.08$ from Figure 4a; c) PSMS/MS spectrum of ions at $\mathrm{m} / \mathrm{z} 399.13$ from Figure 4a; d) PSMS spectrum of authorized glucose in methanol, inset shows molecular structure of glucose; e) PSMS/MS spectrum of sodiated adducts of glucose dimmer [2Glucose $+\mathrm{Na}]^{+}$at $\mathrm{m} / \mathrm{z} 383.08$ from Figure S9d; f) PSMS/MS spectrum of potassiated adduct of glucose dimmer [2Glucose $+\mathrm{K}]^{+}$at $\mathrm{m} / z 399.13$ from Figure S9d. The mass errors of adducts of glucose obtained from tear sample and glucose were inserted in a). 

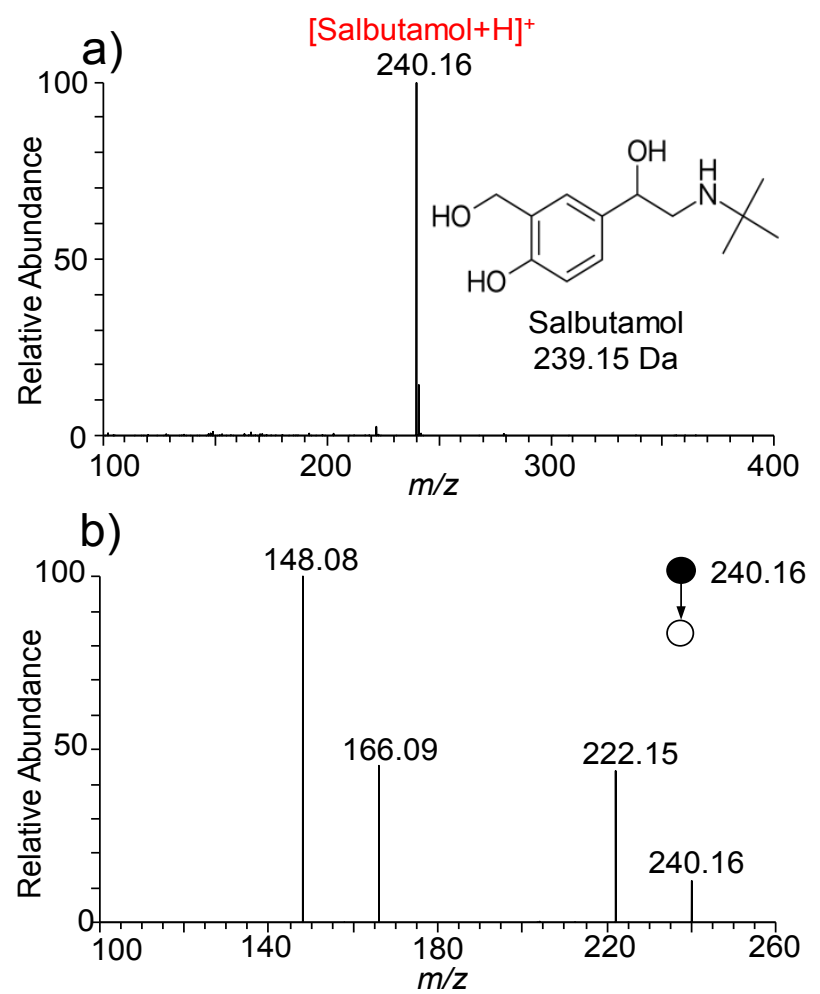

Figure S10. PSMS spectra of standard salbutamol: a) mass spectrum of protonated salbutamol, b) MS/MS spectrum of protonated salbutamol. 

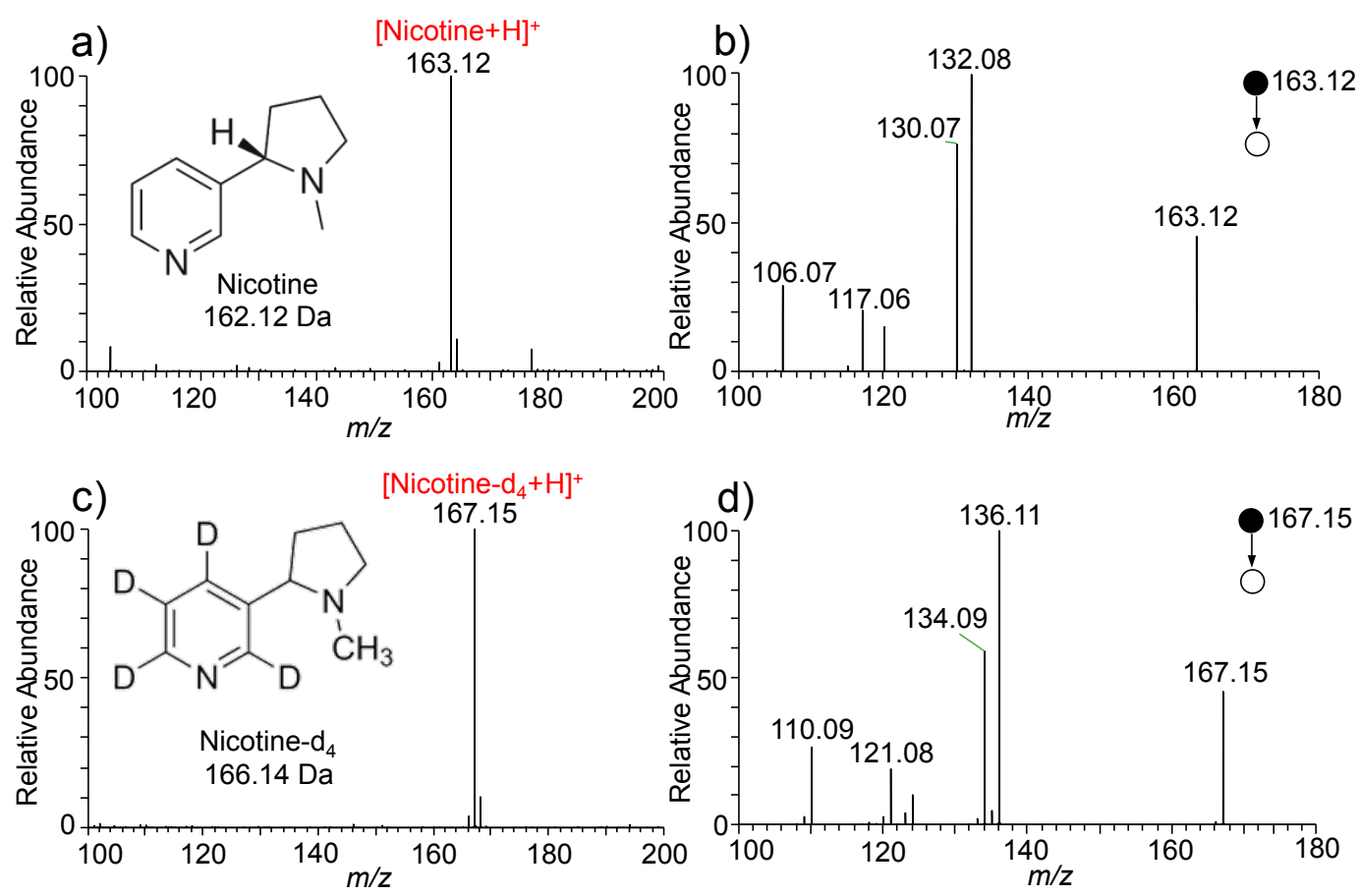

Figure S11. PSMS spectra of authorized nicotine: a) mass spectrum of protonated nicotine, b) MS/MS spectrum of protonated nicotine $(\mathrm{m} / \mathrm{z} 163.12)$, c) mass spectrum of protonated nicotine-d4, d) MS/MS spectrum of protonated nicotine-d4 ( $\mathrm{m} / \mathrm{z}$ 167.15). 

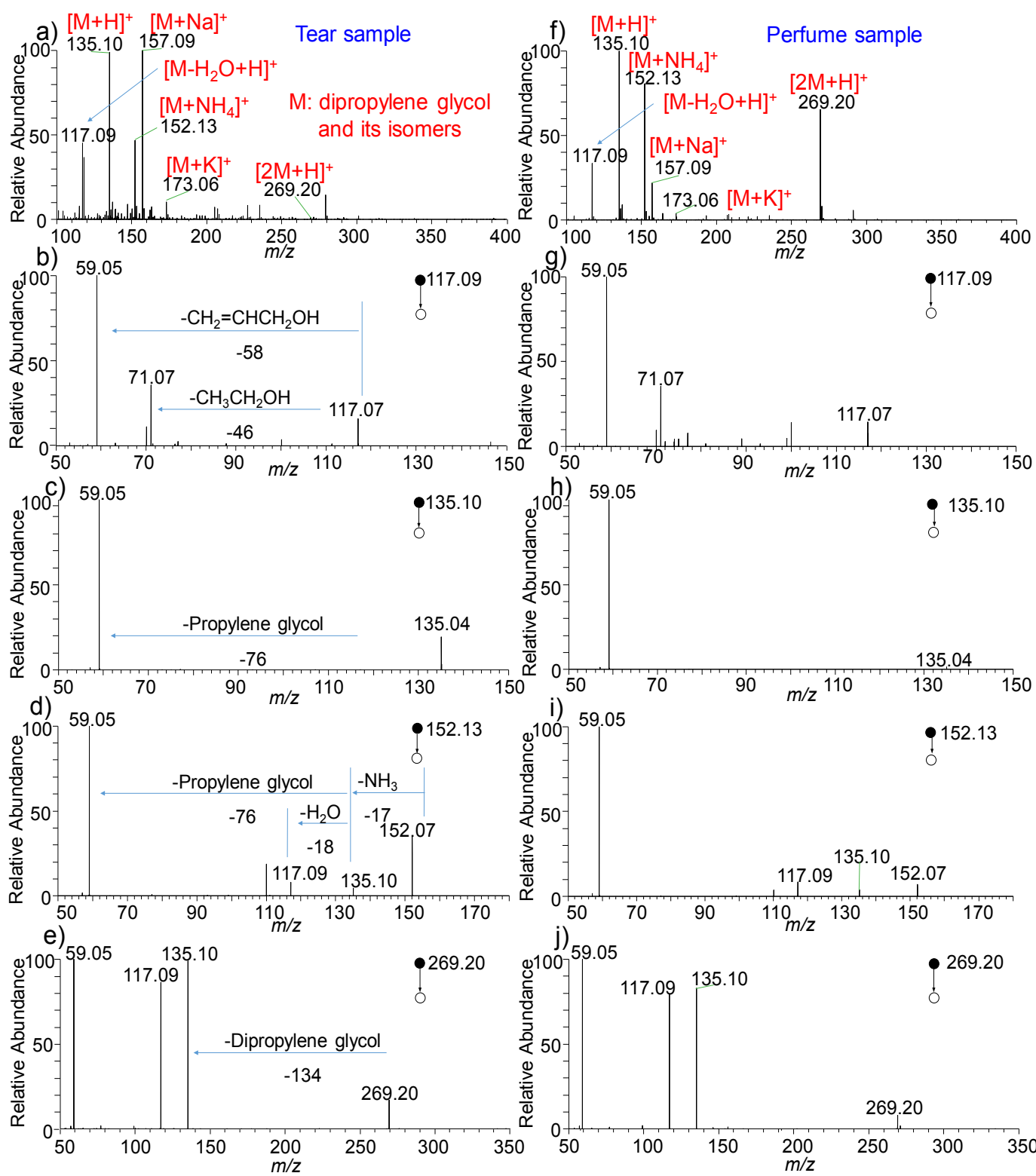

Figure S12. Comparative analysis of perfume-induced tear and perfume by Schirmer PSMS: a-e) Schirmer PSMS analysis of human tear samples exposed to perfume in the air: a) full mass spectrum of tear sample, b) MS/MS spectrum of ions at $m / z 117.09$, c) MS/MS spectrum of ions at $m / z$ 135.13, d) MS/MS spectrum of ions at $m / z$ 152.13, e) MS/MS spectrum of ions at $m / z 269.20$; f-j) direct PSMS analysis of perfume loaded on Schirmer paper (methanol as spray solvent): f) full mass spectrum of perfume sample, g) MS/MS spectrum of ions at $m / z 117.09$, h) MS/MS spectrum of ions at $m / z 135.10$, i) MS/MS spectrum of ions at $m / z 152.13$, j) MS/MS spectrum of ions at $m / z$ 269.20. The identification of adducts $\left(\left[\mathrm{M}+\mathrm{NH}_{4}\right]^{+} \text {at } m / z \text { 152.13, [M+Na }\right]^{+}$at $m / z 157.08,[\mathrm{M}+\mathrm{K}]^{+}$at $\mathrm{m} / \mathrm{z}$ 173.05) by ESI-MS was given in Figure S13. Identification of analytes (dipropylene glycol and its isomers) by GC-MS was given in Figure S14. 

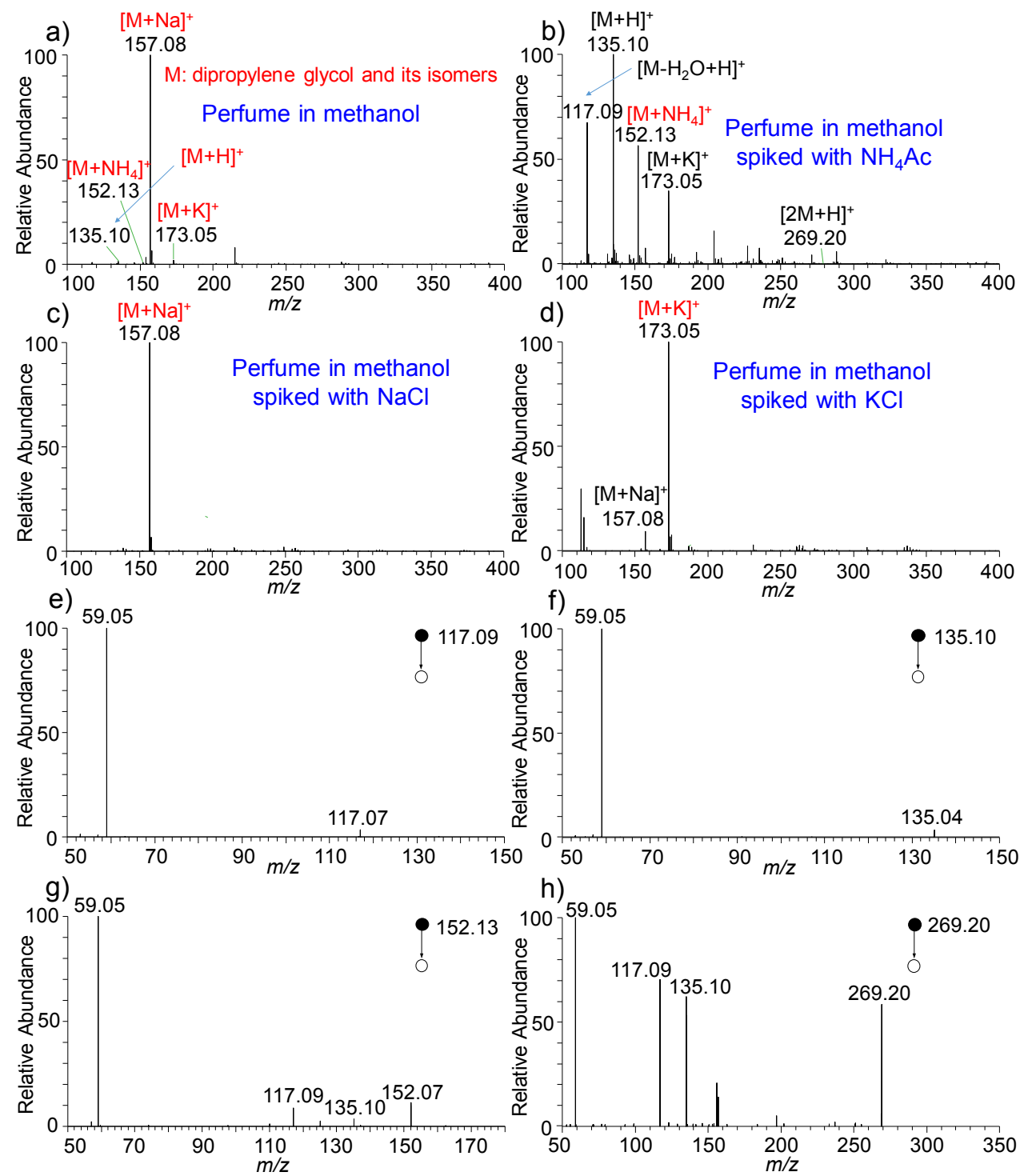

Figure S13. ESI-MS analysis of perfume in methanol solution ( $1.0 \%$, vol $/ \mathrm{vol})$ : a) mass spectrum of perfume in methanol solution; b) mass spectrum of perfume in methanol solution spiked with 1.0 $\mu \mathrm{g} / \mathrm{mL}$ of $\mathrm{NH}_{4} \mathrm{Ac}, \mathrm{c}$ ) mass spectrum of perfume in methanol solution spiked with $0.1 \mu \mathrm{g} / \mathrm{mL}$ of $\mathrm{NaCl}$, d) mass spectrum of perfume in methanol solution spiked with $0.1 \mu \mathrm{g} / \mathrm{mL}$ of $\mathrm{KCl}$, e) $\mathrm{MS} / \mathrm{MS}$ spectrum of the ions at $m / z 117.09$, f) MS/MS spectrum of the ions at $m / z 135.10, \mathrm{~g}$ ) MS/MS spectrum of the ions at $m / z 152.13$, e) MS/MS spectrum of the ions at $m / z 269.20$. The raised peaks at $\mathrm{m} / \mathrm{z} 152.13, \mathrm{~m} / \mathrm{z} 157.08$ and $\mathrm{m} / \mathrm{z} 173.05$ in the mass spectra of spiked samples revealed the formation of analyte adducts by adding $\mathrm{NH}_{4} \mathrm{Ac}, \mathrm{NaCl}$ and $\mathrm{KCl}$, respectively. All the ESI-MS experiments were carried on Orbitrap-MS (Q Exactive, Thermo Fisher Scientific, Bremen, Germany); ESI conditions: ionization voltage at $3.0 \mathrm{kV}$, sheath gas, aux gas and sweep gas were set at 20 bar, 10 bar and $2 \mathrm{~L} / \mathrm{min}$, respectively; MS conditions are same to PSMS. 
a)

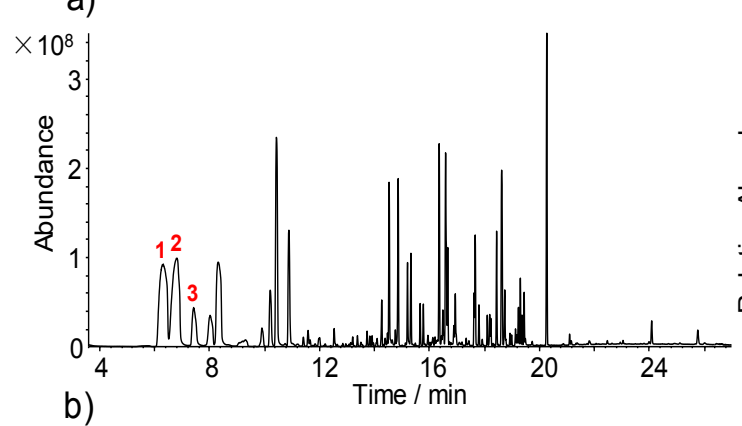

c)

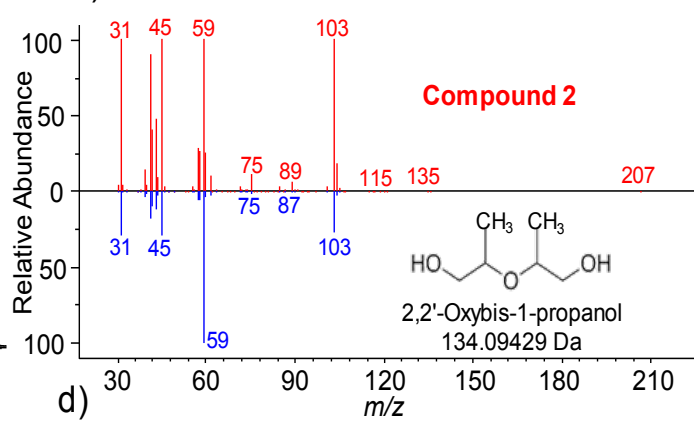

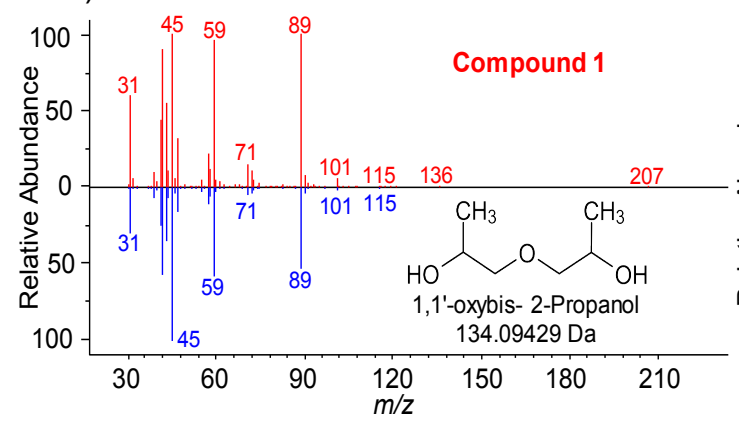

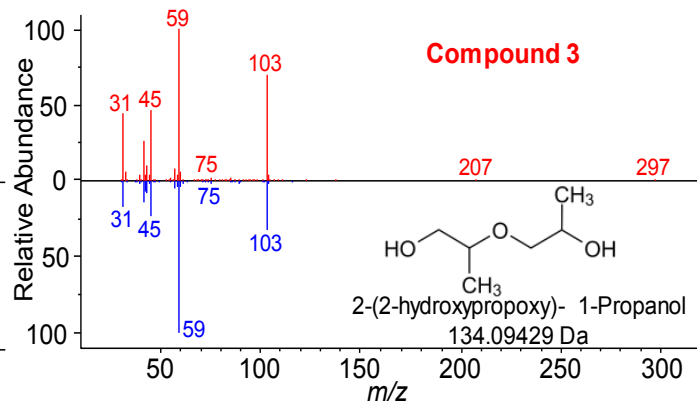

Figure S14. GC-MS analysis of perfume: a) chromatogram of perfume, b) mass spectra of compound 1 (red) and proposed compound (blue), c) mass spectra of compound 2 and proposed compound (blue), d) mass spectra of compound 3 and proposed compound (blue), insert show the molecular structures of proposed compounds. GC-MS experiment was carried on Agilent 7890B GC-5977B inert MSD (Agilent Technologies, Little Fall, NY, USA) under following conditions, column: HP-5MS column $(30 \mathrm{~m} \times 250 \mu \mathrm{m}, 0.25 \mu \mathrm{m}$ film thickness, Agilent Technologies, Palo Alto, CA, USA); carrier gas: He, flowrate: $1.0 \mathrm{~mL} / \mathrm{min}$, temperature of injection: $250^{\circ} \mathrm{C}$; sample volume: $1.0 \mu \mathrm{L}$; temperature programming: $0-5 \mathrm{~min}: 80^{\circ} \mathrm{C} ; 5-22 \mathrm{~min}$ : increased to $250^{\circ} \mathrm{C}$ with a rate of temperature increase at $10^{\circ} \mathrm{C} / \mathrm{min} ; 22-27 \mathrm{~min}: 250^{\circ} \mathrm{C}$; mass spectrometer, ion source: $\mathrm{EI}$, mass range: 30 - $500 \mathrm{amu}$, collision energy: $70 \mathrm{eV}$. 